\title{
Occlusion of the Femoral Artery Induced Fos-like Immunoreactive Neurons in the Lateral Habenular Nucleus Projecting to the Midbrain Periaqueductal Gray in the Rat
}

\author{
By \\ Qiang XIE*, Masahiro ITOH, Takanori MIKI, You Yi DONG and \\ Yoshiki TAKEUCHI \\ Department of Anatomy, Faculty of Medicine, Kagawa Medical University, 1750-1 Ikenobe, Miki-cho, Kita-gun, \\ Kagawa 761-0793, Japan \\ - Received for Publication, July 17, 1998 - \\ Key Words: Lateral habenular nucleus, Periaqueductal gray, FOS-LI, Occlusion, Femoral artery

\begin{abstract}
Summary: Wheat germ agglutinin conjugated horseradish peroxidase (WGA-HRP) injection into the midbrain periaqueductal gray (PAG) resulted in heavy accumulation of retrogradely labeled neurons in the lateral habenular nucleus (LHb) bilaterally. When the left femoral artery was persistently ligated for $2 \mathrm{~h}$, the expression of Fos-like immunoreactivity (FOS-LI) was also found bilaterally in the LHb. In the present study, by combining the retrograde labeling method of injecting WGA-HRP into the PAG and the immunohistochemical staining of the FOS-LI newrons in the LHb induced by occlusion of the femoral artery, it was demonstrated that there are neurons containing both HRP labeling and FOS-LI. These neurons appeared to be located mainly in the medial part and posterior half of the LHb, and constitute about $24 \%$ of the total number of all labeled cells.
\end{abstract}

It was pointed out that the habenular nucleus (Hb) plays an important role as a link between the limbic forebrain structures and midbrain areas $^{5,16,17)}$. In particular, the $\mathrm{LHb}$ has been well known to constitute a relay in the pathway from the nucleus accumbens to the $P_{A G}{ }^{9)}$, and to participate in subserving antinociception ${ }^{18}$. Recently, it has been demonstrated that the LHb neurons respond to noxious input from the peripheral nerve ${ }^{4)}$ and noxious stimulation induces the expression of FOX-LI in the $\mathrm{LHb}^{11)}$. With respect to noxious stimulation of the hindlimb, it is of particular interest that a recent immunohistochemical study also has indicated the expression of FOS-LI in the LHb induced by formalin injection into the unilateral hindpad ${ }^{13)}$. There remain, however, significant lacunae in our knowledge of the effects of ischemia of the hindlimb upon the $\mathrm{Hb}$.

Therefore, the present study was done to elucidate in detail the relationship between LHb neurons having descending projections to the midbrain PAG and FOS-LI neurons in the LHb induced by unilateral occlusion of the femoral artery in the rat.

\section{Materials and Methods}

Twenty-two Wistar rats weighting 200-230 g were used in this study. All surgical procedures were performed under general anesthesia with sodium pentobarbital ( $40 \mathrm{mg} / \mathrm{kg}$ i.p.).

Six rats received a stereotaxic injection of 0.02 $0.03 \mu \mathrm{l}$ of $5-10 \%$ WGA-HRP (Toyobo) into the midbrain PAG using a $1.0 \mu \mathrm{l}$ Hamilton microsyringe attached to a glass micropipette (tip diameter: $30-40 \mu \mathrm{m}$ ). After a survival period of $48 \mathrm{~h}$, the animals were anesthetized and perfused with a fixative containing $1 \%$ paraformaldehyde and $1.25 \%$ glutaraldehyde in $0.1 \mathrm{M} \mathrm{PB}$ (pH 7.4). The brains were removed from the skull immediately and placed into $30 \%$ sucrose in $0.1 \mathrm{M} \mathrm{PB}$ at $4^{\circ} \mathrm{C}$. Serial $50 \mu \mathrm{m}$-thick frozen sections were cut transversely on a microtome and processed for the demonstration of HRP reaction product according to the tetramethyl benzidine (TMB) protocol ${ }^{12)}$.

Six rats were housed individually in a temperature-controlled room. After being anesthetized, the left femoral artery was exposed and the rostral 
portion of the artery was ligated by nylon thread. After persistent ischemia for $2 \mathrm{~h}$, the animals were perfused with $4 \%$ paraformaldehyde in $0.1 \mathrm{M} \mathrm{PB}$ through the ascending aorta. Serial $50 \mu \mathrm{m}$-thick frozen sections were cut through the $\mathrm{Hb}$ and processed for Fos immunohistochemistry according to the method of Hisano et al. ${ }^{7)}$. Briefly, sections were firstly incubated in $10 \%$ normal goat serum (in 0.02 $\mathrm{M}$ phosphate buffer saline with $0.2 \%$ Triton X-100) for $30 \mathrm{~min}$ at room temperature, and then incubated for overnight $\left(\right.$ at $4{ }^{\circ} \mathrm{C}$ ) in polyclonal rabbit antiserum directed against c-Fos protein (Calbiochem.), diluted 1:1000. The sections were washed, exposed to a secondary biotinylated rabbit anti-goat IgG (containing $0.5 \%$ skim milk) for $1 \mathrm{~h}$ at $35^{\circ} \mathrm{C}$, and then processed using the avidin-biotin-complex (ABC) method ${ }^{8}$.

In six rats, $0.02-0.03 \mu$ of $5-10 \%$ WGA-HRP were injected into the PAG. After a survival period of $48 \mathrm{~h}$, the animals were anesthetized again and the left femoral artery was ligated following the same method mentioned above. After $2 \mathrm{~h}$, the animals were perfused with $4 \%$ paraformaldehyde in $0.1 \mathrm{M}$ PB. Sections through the diencephalon were subjected to HRP histochemical reaction by using TMB method, treated with $\mathrm{CoCl}_{2}$ and diaminobenzidine (DAB) to intensify HRP reaction product $^{15}$. Then, the sections were processed for Fos immunohistochemistry.

As a control group, in four rats the left femoral artery was exposed without ligation for $2 \mathrm{~h}$. After perfusion, sections through the $\mathrm{Hb}$ were processed for Fos immnohistochemistry.

\section{Results}

WGA-HRP injection was made into the midbrain PAG. The site of WGA-HRP injection was confirmed as a black mass which was located in the ventral PAG with some diffusion into the dorsal raphe nucleus (DR) as well as the injection shown in Fig. 3B. This injection resulted in retrograde labeling of the LHb bilaterally, while there was no labeling in the medial $\mathrm{Hb}(\mathrm{MHb})$. HRP-labeled neurons, medium in size and oval or triangular in shape, seemed to be confined mainly to the medial part of the LHb with a few distributed to the lateral part (Figs. 1A and 2A).

Occlusion of the left femoral artery resulted in strong bilateral expression of FOS-LI only in the $\mathrm{LHb}$. The number of immunoreactive neurons was much higher than HRP labeling. In addition, it was of interest that these neurons were heavily accumulated in the medial part throughout the LHb although there are some distributed to the lateral part (Figs. 1B and 2B).

WGA-HRP injection into the PAG and ligation of the left femoral artery were made in the same animal. The tracer of enzyme injected into the PAG including the DR was somewhat located eccentrically (Fig. 3B). Then the number of labeled neurons was counted in one rat of the experimental group. This experiment revealed the HRP labeling $(n=349)$ and expression of FOS-LI $(n=509)$ in the LHb. However, the most prominent morphological feature in the present study was that there were neurons containing fine black granules in the cytoplasm with brown-colored nucleus. These neurons labeled with both HRP and Fos protein appeared to be located mainly in the medial part and posterior half of the LHb although there were a few neurons in the lateral part (Figs. 1C and 3A). These neurons were observed to constitute about $24 \%$ ( $n=274)$ of the total number of all labeled cells.

As a control, the left femoral artery was exposed without ligation. This experiment showed very weak expression of FOS-LI in the medial part of the LHb.

\section{Discussion}

The present study demonstrates in the rat that FOS-LI neurons in the $\mathrm{LHb}$, induced by occlusion of the left femoral artery, also project to the midbrain PAG/DR. HRP/FOS double-labeled neurons are characterized by predominant distribution to the medial part of the $\mathrm{LHb}$ with a few distributed to the lateral part. The medial part of the $\mathrm{LHb}$ is regarded as a major relay in the pathway from the limbic forebrain structures to the PAG, raphe nuclei, ventral tegmental area and substantia nigra pars compacta ${ }^{1,9,14)}$. With respect to the functional significance of the $\mathrm{LHb}$, it is reported that the $\mathrm{LHb}$ contains FOS-LI neurons induced by formalin injection into the hindpad ${ }^{13}$ and electrical stimulation of the LHb produces behavioural analgesia $^{3,10)}$. Additionally, the PAG/RD is indicated to be the major site for the endogenous pain control system ${ }^{2,6}$. Considering these anatomical and physiological findings of the LHb and the PAG/ $\mathrm{RD}$, the present study would indicate the LHb to responds to acute ischemia of the hindlimb and subsequently may exert pain modulatory influences on the PAG/DR.

\section{Acknowledgements}

The authors would like to thank Mr. Wakashi Nagata and Mrs Midori Miyawaki for their techni- 
cal assistance.

\section{References}

1) Aghajanian GK and Wang RY. Habenular and other midbrain raphe efferents demonsteated by a modified retrograde tracing technique. Brain Res 1977; 122:229-242.

2) Basbaum AI and Fields HL. Endogenous pain control mechanism: review and hypothesis. Ann Neurol 1978; 4:451-462.

3) Cohen SR and Melzack R. Habenular stimulation produces analgesia in the formalin test. Neurosci Lett 1986; 70:165169.

4) Dafny N and Qian JT. Habenular neuron responses to noxious input are modifed by dorsal raphe stimulation. Neurol Res 1990; 12:117-121.

5) Dong WQ, Wilson OB, Skolnick MH and Dafny N. Hypothalamic, dorsal raphe and external electrical stimulation modulate noxious evoked responses of habenular neurons. Neurosci 1992; 48:933-940.

6) Fields HL and Basbaum AI. Brainstem control of spinal pain-transmission neurons. Annu. Rev Physiol 1978; 40:217-248.

7) Hisano S, Uemura N, Fukui Y, Miki $M$ and Zhang R. Induction of Fos-like immunoreactivity in the rat hypothalamus by an endogenous feeding suppressant (2-buten-4olide). Neurosci Lett 1994; 182:80-82.

8) Hsu SM, Raine $L$ and Fanger $H$. Use of avidin-biotin-peroxidase complex $(\mathrm{ABC})$ in immunoperoxidase techniques: a comparison between $\mathrm{ABC}$ and unlabeled antibody (PAP) procedures. J Histochem Cytochem 1981; 29:577-580.

9) Li YQ, Takada M and Mizuno N. Demonstration of habenular neurons which receive afferent fibers from the nucleus accumbens and send their axons to the midbrain peri- aqueductal gray. Neurosci Lett 1993; 158:55-58.

10) Mahieus $G$ and Benabid AL. Naloxone-reversible analgesia induced by electrical stimulation of the habenular in the rat. Brain Res 1987; 406:118-129.

11) Matsumoto N, Yahata F and Suzuki TA. Tooth pulp stimulation induces c-fos expression in the lateral habenular nucleus of the cat. Neuroreport 1994; 5:2397-2400.

12) Mesulam M-M. Tetramethyl benzidine for horseradish peroxidase neurohistochemistry: a non-carcinogenic blue reaction product with superior sensitivity for visualizing neuronal afferents and efferents. J Histochem Cytochem 1978; 26:106-117.

13) Nagao M, Kamo H, Akiguchi I and Kimura J. Induction of c-Fos-like protein in the lateral habenular nucleus by persistent noxious peripheral stimulation. Neurosci Lett 1993; 151:37-40.

14) Pasquier DA, Anderson C, Forbes WB and Morgane PZ. Horseradish peroxidase tracing of the lateral habenularmidbrain raphe nuclei connections in the rat. Brain Res Bull 1976; 1:443-451.

15) Rey DB, Saper CB and Wainer BH. Stabilization of the tetramethylbenzidine (TMB) reaction product: Application for retrograde and anterograde tracing, and combination with immunohistochemistry. J Histochem Cytochem 1984; 32:1145-1153.

16) Villani L, Zironi $L$ and Guarnieri T. Telencephalohabenulo-interpeduncular connections in the goldfish: a Dil study. Brain Behav Evol 1996; 48:205-212.

17) Yanez $J$ and Anadon $R$. Afferent and efferent connections of the habenula in the rainbow trout (Oncorhynchus mykiss): an indocarbocyanine dye (Dil) study. J Comp Neurol 1996; 372:529-543.

18) Yu LC and Han JS. Habenula as a relay in the descending pathway from nucleus accumbens to periaqueductal grey subserving antinociception. Into J Neurosci 1990; 54:245251. 


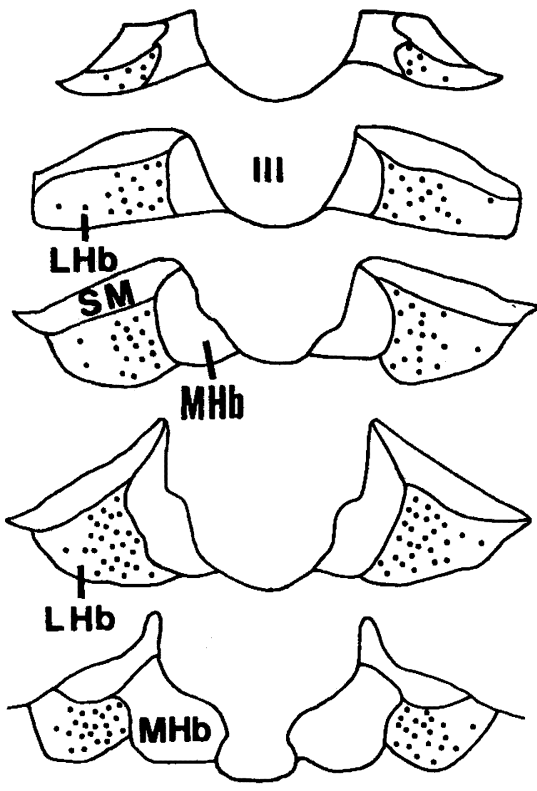

A
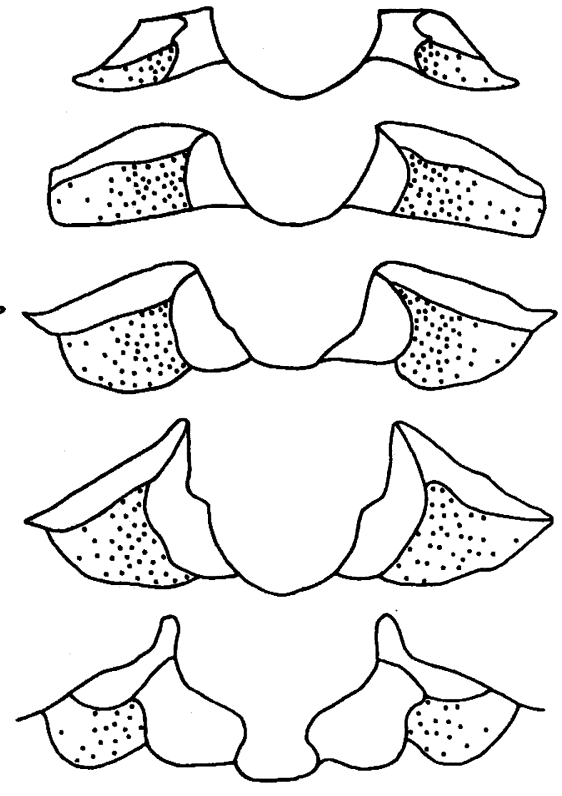

B
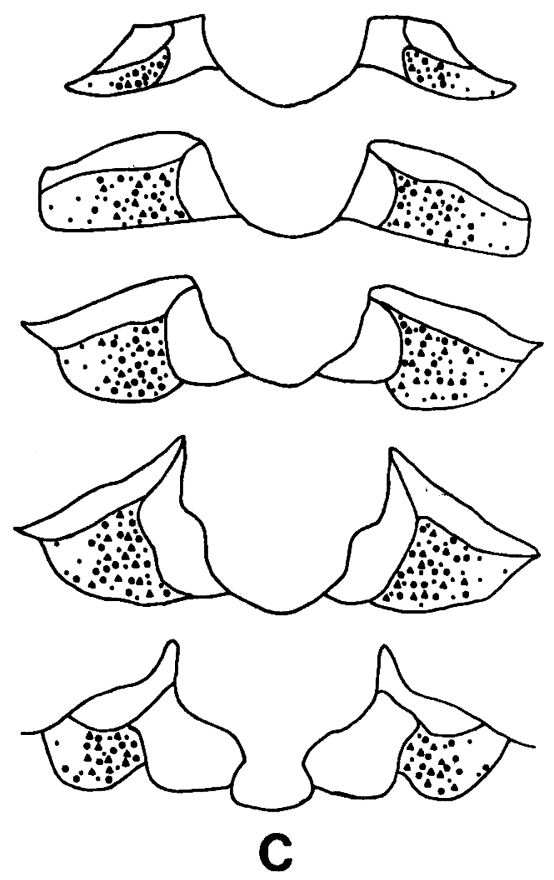

Explanation of Figures

Plate I

Fig. 1. Schematic drawings showing the distrbution of three kinds of labeled neurons in the LHb. Filled circle, small dots and black triangles indicate HRP-labeled $(\bullet)$, FOS-LI $(\bullet)$ and HRP/FOS double-labeled neurons $(\Delta)$, respectively. Note that doublelabeled neurons are confined mainly to the medial part and posterior half of the LHb. Each mark represents two labeled neurons. LHb, Lateral habenular nudeus; MHb, medial habenular nucleus; SM, stria medullaris thalami; III, third ventricle. 
Plate II
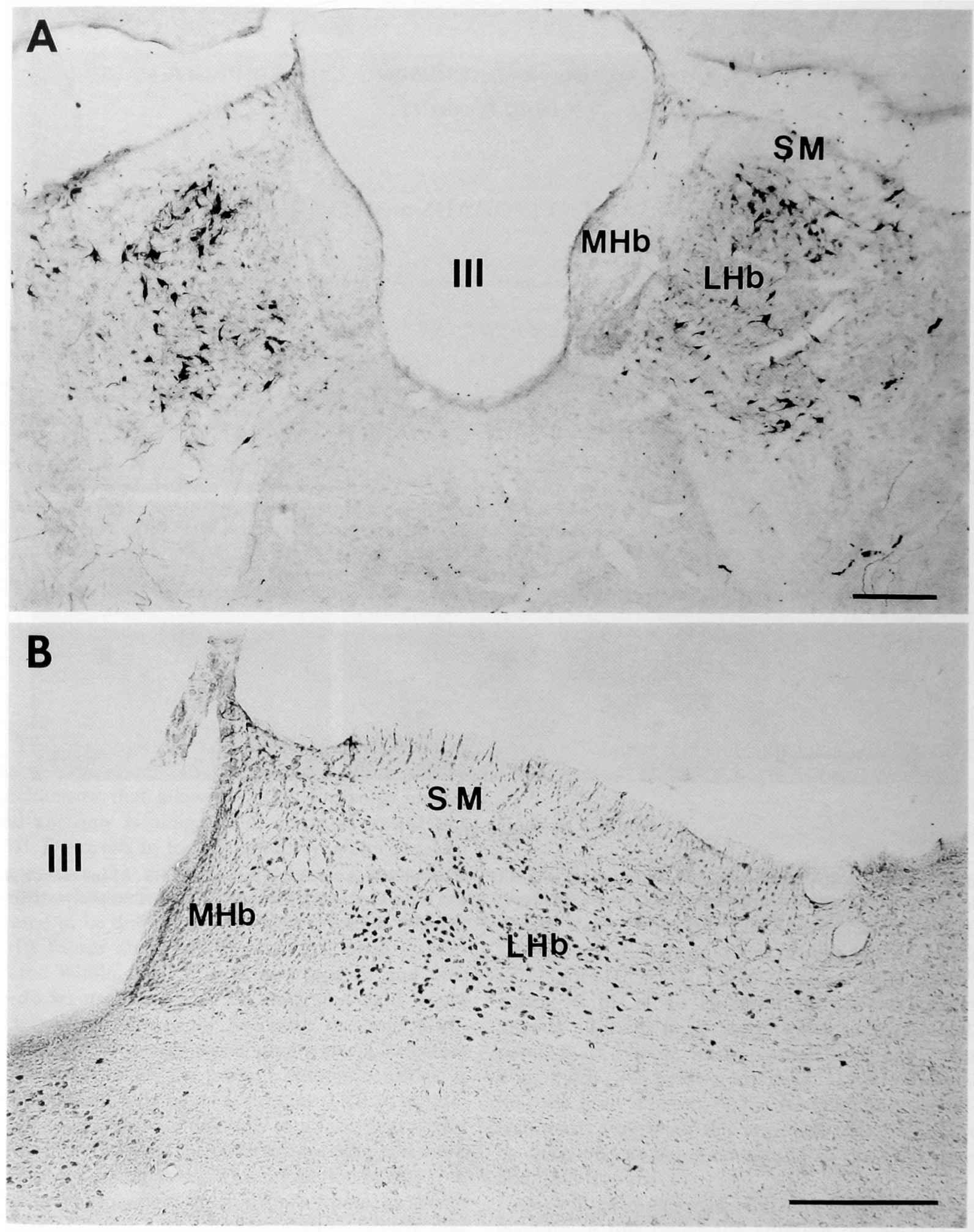

Plate II

Fig. 2. Photomicrographs showing HRP-labeled (A) and FOS-LI neurons (B) in the LHb. Note that neurons labeled with HRP and fos protein are also found mainly in the medial part of the LHb. LHb, lateral habenular nucleus; MHb, medial habenular nucleus; SM, stria medullaris thalami; III, third ventricle. Scale bars $=200 \mu \mathrm{m}$ in A and B. 
Plate III

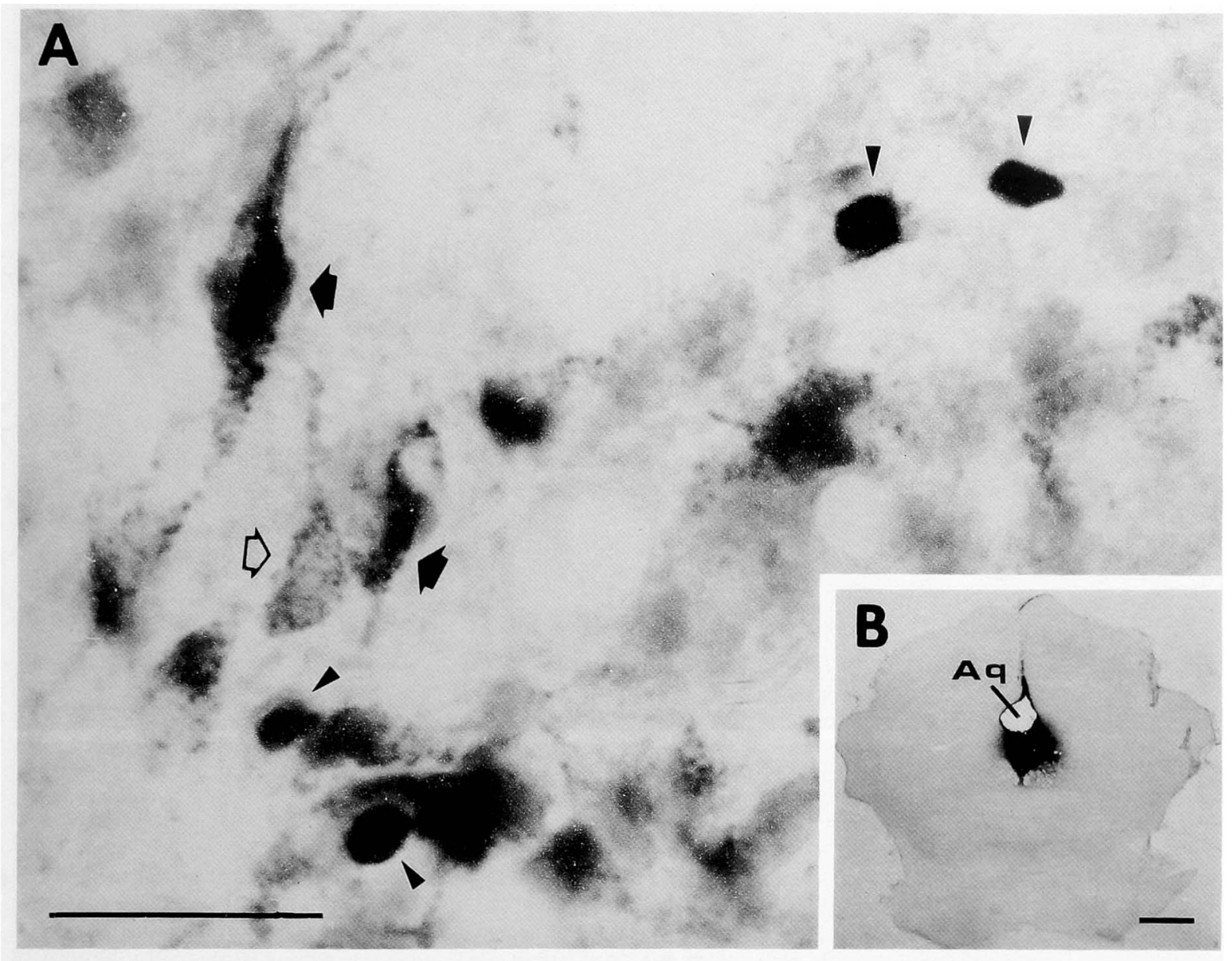

Plate III

Fig. 3. Photomicrographs showing neurons labeled with both HRP and Fos protein in the medial part of the LHb (A) and the site of WGA-HRP injection into the the PAG (B). Solid and open arrows and arrowheads indicate HRP/FOS double-labeled, HRPlabeled and FOS-LI neurons, respectively. Aq, aqueductus cerebri. Scale bars $=50 \mu \mathrm{m}$ in $\mathrm{A}$ and $1 \mathrm{~mm}$ in $\mathrm{B}$. 